\title{
Philosophiques
}

\section{Le projet de loi 50 et la place des droits de l'homme dans le système juridique}

\section{Claude Panaccio}

Volume 2, numéro 2, octobre 1975

URI : https://id.erudit.org/iderudit/203039ar

DOI : https://doi.org/10.7202/203039ar

Aller au sommaire du numéro

Éditeur(s)

Société de philosophie du Québec

ISSN

0316-2923 (imprimé)

1492-1391 (numérique)

Découvrir la revue

Citer ce document

Panaccio, C. (1975). Le projet de loi 50 et la place des droits de l'homme dans le système juridique. Philosophiques, 2(2), 364-375.

https://doi.org/10.7202/203039ar d'utilisation que vous pouvez consulter en ligne.

https://apropos.erudit.org/fr/usagers/politique-dutilisation/ 


\title{
LE PROJET DE LOI 50 ET LA PLACE DES DROITS DE L'HOMME DANS LE SYSTÈME JURIDIQUE
}

\author{
par Claude Panaccio
}

Le principal débat public auquel a donné lieu la présentation par le Gouvernement du Québec du projet de loi 50 sur les libertés et les droits de la personne portait sur la place exacte d'une telle loi dans le système juridique où elle vient s'inscrire. L'article 45 du projet de loi $^{1}$ stipulait entre autres :

«La présente Charte (...) ne doit pas, non plus, s'interpréter de manière à augmenter, restreindre ou modifier la portée de toute disposition de la loi; toutefois, si un doute surgit dans l'interprétation de telle disposition, il est tranché dans le sens indiqué par la présente Charte."

Plusieurs organismes, dont la Ligue des Droits de l'homme, la Fédération des Travailleurs du Québec, le Réseau d'Action et d'Information pour les femmes (R.A.I.F.), le Barreau du Québec, ont fait remarquer, dans les mémoires qu'ils ont soumis à la commission parlementaire, qu'une telle clause limitait indûment l'importance de cette éventuelle Charte des droits de la personne, qu'elle risquait même de la transformer en un simple code d'interprétation des autres lois, qu'idéalement il aurait fallu en faire une loi fondamentale à laquelle eût été subordonnée toute la législation québécoise, et que, si cette subordination présente vraiment pour les lois déjà promulguées d'insurmontables difficultés pratiques, on aurait dû au minimum prévoir que toute loi à venir serait soumise à la nécessité légale d'être compatible avec ladite Charte. Comme le dit de façon laconique le mémoire du R.A.I.F.: "Une Charte qui n’a pas préséance sur les autres lois n'est plus une Charte. ${ }^{2}$

1. Toutes les références au projet de loi 50 qu'on trouvera ici renvoient au texte soumis en première lecture. Que ce texte ait été modifié par la suite ne change d'ailleurs rien au fond du débat.

2. Loi sur des droits et libertés de la personne, mémoire soumis par le Réseau d'action et d'information pour les femmes à la commission parlementaire de la justice, janvier 1975, p. 22. 
Qu'on la conçoive comme logique du discours juridique, comme réflexion sur les rapports du droit et de la morale ou comme critique des appareils idéologiques, la philosophie du droit est ici directement concernée; et c'est à tous ces niveaux à la fois que je voudrais maintenant reprendre à nouveaux frais la question de la place des droits de l'Homme dans un système juridique comme le nôtre, en illustrant mon propos par l'exemple privilégié (du moins pour nous) de cette récente discussion d'actualité.

J'aborderai le problème par le biais de deux sous-questions :

$1^{\circ}$ Les droits de l'Homme, lorsqu'ils s'inscrivent dans un système législatif particulier, doivent-ils être conçus comme la projection à l'intérieur de ce système de certains droits naturels déjà inscrits dans l'être même de l'homme, ou comme de pures conventions plus ou moins arbitraires sur le plan logique ou sur le plan ontologique?

$2^{\circ}$ Si l'on accepte la seconde interprétation - que pour ma part je préférerai ici -, la Charte des droits de l'Homme peutelle vraiment servir de «fondement » à tout le système législatif?

\section{1. - Droit naturel et convention juridique}

La première de ces questions me paraît la moins intéressante dans les circonstances. D'abord, le débat qui oppose les tenants du droit naturel et les positivistes dure déjà depuis suffisamment longtemps pour qu'il soit inutile de le reprendre ici depuis le début. Ensuite, cette discussion entraîne inévitablement celui qui s'y engage à déboucher aussitôt sur de vieux problèmes épistémologiques, qui, certes, sont encore loin de faire l'unanimité, mais qui ont tout de même beaucoup perdu de leur intérêt. La position naturaliste, en effet, implique directement la notion typiquement métaphysique d'une nature humaine normative, capable par elle-même d'imposer à l'action collective ou individuelle des impératifs catégoriques et universels, notion qui, on le sait, a fait depuis plusieurs années déjà l'objet de nombreuses critiques extrêmement sévères de la part de mouvements philosophiques aussi différents les uns des autres que le positivisme 
logique, l'existentialisme sartien et le marxisme, et je n'ai pas l'intention de reprendre ici ces critiques dans le détail.

La question néanmoins, quel qu'en soit le caractère éculé, demeure pertinente à notre propos. Si les naturalistes devaient avoir raison, la place idéale d'une Charte des droits de l'Homme dans un système juridique paraîtrait - du moins à première vue - relativement claire. Sa fonction consisterait simplement à enraciner ledit système dans la morale naturelle en donnant aux principes de cette morale une formulation juridique adaptée aux circonstances sociales et historiques. Auquel cas bien sûr la Charte en question devrait automatiquement être prioritaire par rapport à toute autre loi. Si en effet une législation quelconque, passée ou future, devait s'avérer incompatible avec la Charte des droits de l'Homme, elle serait du même coup incompatible avec la morale naturelle (pour peu du moins que le texte de la Charte corresponde vraiment aux principes éthiques inscrits dans l'être). Admettre ce point serait par définition admettre qu'il s'agit d'une mauvaise loi. Il y aurait contradiction nette à affirmer, d'une part, que les droits de l'Homme correspondent à des normes naturelles de l'action moralement bonne et à considérer, d'autre part, comme acceptable telle loi qui serait en même temps reconnue incompatible avec ces droits.

Dans la mesure exacte où, en affirmant dans son préambule que «tout être humain possède des droits et des libertés intrinsèques», le projet de loi 50 souscrit à la métaphysique naturaliste, il n'échappe pas à cette contradiction lorsqu'à l'article 45 il laisse ouverte la possibilité de lois qui soient à la fois acceptables et incompatibles avec la Charte. Sur ce plan, les critiques, qui pour la plupart se situent également à l'intérieur de la métaphysique naturaliste, ont donc parfaitement raison.

La contradiction cependant demande à être analysée de plus près. Ce qu'elle révèle en effet, c'est moins la faiblesse du législateur en matière de logique que l'existence d'une tension entre la pratique réelle du droit et l'idéologie officielle qui lui sert de justification. Si, dans le préambule et dans les discours politiques qui présentent ou qui discutent le projet de loi, l'idéologie naturaliste demeure dominante, il reste que dans le texte lui-même et dans l'acte législatif qui l'institue en loi, tout se passe comme s'il 
s'agissait d'une simple convention. Entre l'affirmation du préambule que je citais plus haut et l'article 1 , par exemple, qui stipule que «tout être humain a droit à la vie ainsi qu’à la sûreté et à la liberté de sa personne », il n'y a pas un simple lien de redoublement ou de spécification; il y a toute la distance qui sépare un énoncé métaphysique d'un performatif juridique. Le second en tout cas garde toute sa force, quelle que soit la valeur du premier, et l'on sait depuis longtemps, surtout au Québec, que le texte d'une loi peut facilement en contredire le préambule ou le titre sans que le système juridique lui-même ne soit contradictoire pour autant. Le préambule, le titre, les discours officiels sont là pour mieux faire passer une loi auprès de l'opinion publique et non pas pour lui servir de prémisses. À vrai dire, même les mémoires soumis à la commission parlementaire, lorsqu'ils abandonnent les déclarations de principe - souvent naturalistes -, demandent au législateur d'introduire dans le texte de son projet des choses qu'on peut difficilement considérer comme des droits naturels : le droit à la syndicalisation, le droit au travail rémunéré, le droit de grève, le droit à l'information, le droit à l'anesthésie dans les interventions chirurgicales, etc...

On aura beau dire qu'il ne s'agit là de rien d'autre que de moyens historiquement situés pour favoriser le respect des véritables droits naturels tels que le droit à la liberté, à l'égalité, à la propriété, etc..., il faut bien se rendre compte que dans le texte même de la loi, il n'y a pas entre la promulgation des droits fondamentaux et celle des droits historiquement relatifs une relation de prémisses à conclusions ou de fins à moyens, mais une simple juxtaposition d'un article par rapport à un autre. Ainsi le chapitre I du projet de loi 50 , intitulé «Libertés et droits fondamentaux », comporte aussi bien l'affirmation du droit à la vie, (Art. 1) que celle du droit au respect du secret professionnel (Art. 10) ou que l'interdiction de refuser par discrimination de conclure un bail (Art. 14). Le texte légal n'établit par lui-même aucune opposition pertinente entre ce qui est naturel et ce qui est historique.

Comment d'ailleurs en serait-il autrement? $\grave{A}$ supposer même - ce dont je doute absolument - que la thèse naturaliste soit juste, il faudrait néanmoins reconnaître que nous ne dispo- 
sons, pour procéder à l'inventaire des valeurs et des normes inscrites dans la nature, d'aucune procédure généralement admise qui puisse être légalement reconnue comme telle. Dans les faits, on ne justifie pas l'introduction dans une Charte de tel ou tel droit en en prouvant rigoureusement le caractère naturel (il faudrait alors confier la préparation des lois à des comités de métaphysiciens!), mais en montrant qu'il rejoint le sentiment de la majorité, ou que son adoption serait bénéfique pour l'ordre social, ou en invoquant toute autre raison - quelle qu'en soit la nature - susceptible de rallier l'opinion publique.

De toute manière, le principal argument de ceux qui s'opposent au naturalisme me paraît absolument probant : si un droit existait de façon naturelle, il ne serait plus un droit mais un fait, et d'un état de fait on ne peut pas déduire une norme ${ }^{3}$. Certes, cet argument n'est pas accepté par tout le monde. Même sa formulation proprement logique ( d'un ou de plusieurs jugements de fait, on ne peut inférer un jugement de valeur») fait encore l'objet de nombreuses discussions. Mais, outre que les tentatives de réfutation qu'on en a proposées me paraissent le plus souvent assez fumeuses ${ }^{4}$, il faut voir que les critiques les plus sérieuses, fussent-elles fondées, ne conduiraient pas du tout à la réhabilitation d'une notion comme celle de droit naturel. Searle, par exemple, pense avoir trouvé la recette pour générer un nombre indéfini de contre-exemples où il serait parfaitement licite de

3. Depuis G.E. MOORE (Princspia Ethica, Cambridge, Cambridge University Press, 1903), cet argument a été maintes fois repris dans le cadre des discussions sur la logique du discours moral (cf. en particulier: HARE, R.M., The Language of Morals, Oxford, Oxford University Press, 1968). En philosophie du droit proprement dite, on le trouve notamment chez G. KALINOWSKI (cf. par exemple: "Obligation dérivée et logique déontique relationnelle», in Notre-Dame Journal of Formal Logic, V, 1964, p. 186; et «Contribution à la critique de la philosophie existentialiste du droit», in Arcbives de pbilosopbie du droit, IX, 1964, p. 268) et chez H. KELSEN (cf. par exemple: «Justice et droit naturel», in Annales de philosopbie politique, III, 1959, p. 68-69).

4. Cf. par exemple: GARDIES J.L., Essai sur les fondements a priori de la rationalité morale et juridique, Paris, Librairie Générale de Droit et de Jurisprudence, 1972, p. 13-32. Gardies soutient la thèse ontologico-phénoménologique, que pour ma part je trouve inintelligible, selon laquelle il y aurait déjà dans l'être un certain devoir-être sans en fournir d'autre preuve qu'une critique facile de la notion de fait, ce qui, je crois, passe à côté de la question. 
déduire un jugement de valeur à partir de prémisses factuelles ${ }^{5}$, mais sa démonstration, qu'elle soit valable ou non, ne fonctionne qu'à la condition expresse que les prémisses en question expriment un type bien particulier de faits que Searle appelle les «faits institutionnels » ceux dont l'existence est elle-même tributaire d'une institutionnalisation humaine déjà réglementée (le cas de la promesse, par exemple, ou celui d'un événement interne au jeu de baseball ou à tout autre jeu). L'argument de Searle ne concerne donc nullement l'existence des droits dits naturels.

Tout compte fait, il paraît très difficile sur le plan théorique de continuer à considérer les Chartes des droits de l'Homme comme des échos plus ou moins fidèles de la voix caverneuse de la nature humaine. Dans la mesure surtout où, de toute manière, la pratique même du droit ne présuppose rien de tel, mieux vaut, pour des raisons de simplicité et d'intelligibilité, considérer qu'une Charte des droits de l'Homme n'est rien d'autre qu'une convention historiquement située, édictée par des hommes ou par des institutions pour des raisons qui peuvent être fort variables d'un cas à l'autre et qui, en tout cas, n'ont généralement rien de métaphysique (sauf encore une fois au niveau de l'idéologie officielle au sein de laquelle on justifie ou on critique une telle loi).

\section{2. - Loi fondamentale ou loi particulière ?}

Si ce point est admis, la question originale peut maintenant être reformulée: quelle est la place qui revient ou qui peut revenir à cette convention particulière dans le système des conventions juridiques d'une société comme la nôtre? En particulier, quel sens y aurait-il à en faire le postulat de tout le système? Dans cette perspective conventionnaliste, - ou en tout cas plus relativiste, - les tenants de la thèse selon laquelle la Charte des droits de l'Homme devrait être une loi fondamentale considèrent qu'une telle loi est l'occasion d'expliciter les valeurs de base auxquelles adhère une société donnée à un moment donné, quelles que soient par ailleurs les raisons ou les causes de cette adhésion et sans préjuger du caractère «naturel » ou * absolu» de ces valeurs.

5. Cf. SEARIE J.R., Speech Acts, Cambridge, Cambridge University Press, 1969. 
Relativement à la place exacte de la Charte dans le système des lois, cette thèse peut prendre deux formes différentes. La version la plus stricte consiste à concevoir le système juridique sur le modèle des systèmes formels. La Charte des droits y jouerait alors le rôle des axiomes dont tout le reste doit pouvoir être déduit $^{6}$; et si certaines lois résistaient encore à la possibilité d'être directement ou indirectement déduites de ces axiomes, il faudrait, à tout le moins, qu'elles ne fussent pas incompatibles avec eux. L'autre version, plus pragmatiste, voit dans la Charte quelque chose comme un guide pour la vie sociale et juridique, l'énoncé de certains principes généraux auxquels, autant que faire se peut, la société essaie de se conformer ${ }^{7}$; les liens logiques qui unissent les lois ordinaires à la Charte sont ici beaucoup plus lâches, et si certaines incohérences subsistent, on essaiera de les corriger à la longue en tenant compte de ceci qu'un système juridique n'est précisément pas un système formel, mais s'édifie lentement au fil des circonstances sur la base d'un certain nombre de principes directeurs.

Mais l'une et l'autre position ont quelque chose d'utopique. La première oublie certainement, comme le lui font remarquer les tenants de la seconde, qu'aucun système juridique actuel n'est véritablement formalisable et que, d'ailleurs, même si cela était possible, il n'y aurait peut-être pas avantage à le faire d'un seul coup. C'est ainsi, par exemple, pour revenir au cas du projet de

6. C'est, par exemple, une fonction de ce genre que KELSEN assigne à ce qu'il appelle la «constitution»; cf. Pure Theory of Law, Trad. anglaise de M. Knight, Los Angeles, University of California Press, 1967, p. 221-222: «The legal order is not a system of coordinated norms of equal level, but a hierarchy of different levels of legal norm. Its unity is brought about by the connection that results from the fact that the validity of a norm, created according to another norm, rests on that other norm, whose creation in turn, is determined by a third one. This is a regression that ultimately ends up in the presupposed basic norm. This basic norm, therefore, is the highest reason for the validity of the norms, one created in conformity with another, thus forming a legal order in its hierarchical structure. Considering, to begin with, only a national legal order, the constitution represents the highest level of positive law».

7. Cf. par exemple : Chaïm PERELMAN, «Peut-on fonder les droits de l'homme? 》 in Droit, morale et pbilosopbie, Paris, Librairie générale de droit et de jurisprudence, 1968, pp. 57-64; ou encore: Fernand DUMONT, «Droit et utopie: la Déclaration des droits de l'homme» in Chantiers, Montréal, Hurtubise HMH, 1973, pp. 229-242. 
loi 50, que ceux qui s'opposent à ce que son adoption provoque une révision radicale de tout le système font remarquer que, la coutume étant une dimension importante de l'ordre social et de la vie juridique, une telle révision, loin de clarifier les relations sociales, engendrerait à court et à moyen terme une confusion et une insécurité fort inquiétantes :

«si ce projet de loi devait prévaloir sur toutes les lois présentement en vigueur, lit-on dans le mémoire du Comité de Législation de la Chambre des Notaires, il en résulterait un climat d'incertitude nuisible à la sécurité des conventions. Il est à prévoir que des jugernents d'équité viendraient semer le doute sur la validité d'articles par ailleurs clairs et précis et des interprétations jurisprudentielles jusque là bien établies. L'ambiguïté qui en résulterait serait néfaste à tous les justiciables " 8 .

La position pragmatiste, quant à elle, oublie qu'en l'absence de règles précises d'application et d'interprétation, des principes aussi généraux et abstraits que le droit à la liberté, le droit à la vie, le droit à l'égalité n'ont, en définitive, aucun impact véritable et qu'ils ne peuvent pas vraiment servir de façon efficace à guider les relations sociales et l'activité juridique. Plusieurs commentateurs ont déjà fait remarquer, à propos de la Déclaration universelle des Droits de l'Homme adcptée en 1948 par l'Assemblée Générale des Nations Unies, que seule l'extrême imprécision du texte a pu permettre aux représentants de régimes politiques et économiques aussi différents les uns des autres de s'entendre à son propos.

Il n'est qu'à voir, d'ailleurs, dans les débats actuels qui entourent le projet de loi 50 comment les différents mémoires soumis à la commission parlementaire utilisent tous à l'appui de leur argumentation les mêmes principes d'égalité et de liberté pour revendiquer les choses les plus diverses et les moins conciliables. Il est sans doute significatif de voir notamment la Fédération des Travailleurs du Québec et la Chambre de Commerce

8. Projet de loi 50 : loi sur les droits et libertés de la personne, mémoire soumis par le comité de législation de la chambre des notaires à la commission parlementaire de la justice, p. 9; on trouve aussi un argument semblable dans le Mémoire de la Commission des Écoles Catholiques de Montréal à la Commission Parlementaire de la Justice, janvier 1975, p. 1-2. 
de la Province de Québec s'entendre sans difficultés sur les principes généraux; mais lorsque vient le moment d'en tirer des conclusions pratiques, la F.T.Q. fait remarquer, par exemple, que l'égalité sociale et économique n'est pas suffisamment assurée par la loi ${ }^{9}$, ou que la liberté de grève est encore trop restreinte ${ }^{10}$, alors que la Chambre de Commerce observe, pour sa part, que le projet de loi 50 viole lui-même le principe d'égalité devant la loi en accordant plus de facilités à un éventuel plaignant (celui qui s'estimerait lésé dans ses droits fondamentaux) qu'à la personne ou à l'organisme contre qui la plainte serait adressée ${ }^{11}$, ou encore que le droit à la libre disposition de la propriété est contrecarré dans la législation actuelle par les règlements municipaux de zonage ou par la loi des biens culturels qui interdit de transporter hors du Québec des biens reconnus sans la permission du ministre ${ }^{12}$.

La généralité même des principes en cause autorise à les utiliser aussi bien pour défendre l'image d'une société fondée sur l'initiative privée que pour prôner une conception plus socialisante, voire même carrément communiste. L'introduction inévitable, dans la formulation de ces principes, de termes évaluatifs émotivement très chargés et cognitivement très pauvres (liberté, égalité, personne humaine...) compromet sérieusement cette fonction de guide fondamental des relations sociales que d'aucuns voudraient voir assumée par la Charte. Le caractère inopérant de ces principes vient de ce qu'ils ne tiennent aucun compte de la réalité sociale elle-même qui, du moins dans l'état actuel des choses, est essentiellement de nature conflictuelle.

Mais il y a encore plus : au niveau de la pratique juridique, non seulement les principes basés sur les droits de l'Homme sont-ils inefficaces pour orienter l'ensemble de la législation, mais dans beaucoup de cas les lois, loin de pouvoir être considé-

9. Cf. Mémoire soumis par la Fédération des Travailleurs du Québec à la Commission Parlementaire chargée d'étudier le projet de loi 50 ou «Charte des droits $\gg$, janvier 1975 , p. 4.

10. Id. p. 5.

11. Cf. Mémoire sur le projet de loi 50 «Loi sur les droits et libertés de la personne, » soumis par la Chambre de Commerce de la Province de Québec à la commission parlementaire de la justice, janvier 1975, p. 4.

12. Id. p. 9. 
rées comme des conclusions qui se dégageraient directement ou indirectement de ces principes, apparaissent en réalité comme des exceptions aux droits de l'Homme. Ainsi l'article 1 de la loi 50 affirme que «tout être humain a droit à la vie ainsi qu'à la sûreté et à la liberté de sa personne », mais il est implicitement évident que ceci ne s'appliquera jamais aux cas où la loi ellemême condamne quelqu'un à la mort ou à la prison. De même, l'article 2 assure que «toute personne dont la vie est en péril a droit au secours », mais évidemment cet article n'autorise pas à essayer de faire évader un condamné à mort ou à intervenir pour empêcher un policier d'abattre un voleur. L'article 3 précise que «tout être humain a droit à la liberté d'expression», mais on sait bien que ce «droit» n'existera plus le jour où la loi jugera que les opinions de l'être humain en question sont subversives. L'article 7 pose que «la demeure est inviolable», mais ceci n'empêche jamais aucun juge de délivrer des mandats de perquisition.

On pourrait poursuivre encore longtemps cette énumération, mais contentons-nous d'un dernier exemple, sans doute le plus frappant de tous, celui de l'article 10 : «Toutes les personnes sont égales devant la loi. » Qu'est-ce que cela signifie? À première vue, que les lois sont les mêmes pour tous. Mais à y regarder de plus près, les choses ne sont pas si simples : certaines lois ne s'appliquent qu'aux propriétaires, d'autres aux locataires, certaines concernent uniquement les Indiens, d'autres uniquement les fermiers, d'autres uniquement les policiers, ou les syndiqués, ou les automobilistes, ou les enfants, etc... Et parmi ces différences obligatoirement reconnues pertinentes par la loi, certaines relèvent de prédicats qui sont rattachés aux personnes en question de façon absolument contingente (comme le prédicat « avoir commis un vol », mais d'autres leur collent plus intimement à la peau (comme le fait d'être Indien, d'être né dans tel pays, ou d'appartenir à tel sexe).

En fait, un système législatif ne peut fonctionner qu'en instituant des différences. Même l'interprétation selon laquelle la véritable égalité consiste à traiter chacun selon sa situation propre et selon ses mérites n'échappe pas à cette difficulté, puisqu'il faut bien reconnaître que c'est la loi elle-même qui, en l'occurrence, 
définit ce qu'est une «situation propre » et ce qui est un «mérite » : il faudrait accepter d'appeler «égalité » l'institution d'un jeu de différences. Ceci étant reconnu, on ne peut interpréter le principe d'égalité devant la loi que comme un principe de clôture de la liste des différences pertinentes qui n'affirme en réalité rien d'autre que ceci : seules les différences expressément reconnues par la loi entraînent devant les tribunaux des différences de traitement et toute opposition qui ne serait pas inscrite dans le texte législatif doit être considérée comme non pertinente. Ainsi compris, - et cela semble bien être l'interprétation qui se dégage de la logique interne du discours juridique, - le principe d'égalité ne saurait plus apparaître comme l'une des prémisses du système juridique: il n'est qu'une stipulation logiquement subordonnée au reste du système.

La même interprétation s'applique mutatis mutandis aux autres droits dits fondamentaux. Introduits dans le texte juridique, ils constituent des clauses de clôture par rapport aux promulgations législatives, mais ce qu'ils limitent ainsi, ce ne sont pas vraiment les lois elle-mêmes, ce sont plutôt les comportements de ceux qui ne représentent pas la loi: nul autre que la loi ne peut priver quelqu'un de sa liberté, nul autre que la loi ne peut donner la mort, et ainsi de suite. Au fond, l'article 45 du projet de la loi 50 respecte sans doute plus la logique du système juridique que la position de ceux qui voudraient faire de la Charte une « loi fondamentale» régissant l'ensemble des autres lois.

\section{3. - La dimension idéologique}

Certes, il y a là comme un paradoxe. Car, malgré l'article 45 , il est certain que la justification officielle du projet de loi 50 (le préambule, les discours de présentation, etc...), de même que le vocabulaire qu'il utilise (liberté, égalité, droits de la personne, etc...) semblent bien lui attribuer une toute autre portée, qui lui viendrait d'un lien bien spécial qu'il entretiendrait avec les valeurs les plus profondes de l'humanité. $\mathrm{Si}$, quittant le plan de la froide logique juridique, on se laisse inspirer par les connotations vastes et mystérieuses qui pullulent aussi bien dans le texte même du projet de loi que dans les discours qui l'entourent, la Charte des droits de l'Homme nous apparaîtra comme le porte- 
parole dans le système juridique de la Morale (avec un grand « $\mathrm{M} »)$ la plus fondamentale.

La question est de savoir comment il est possible que cette loi joue ainsi un tel double jeu : du strict point de vue de son contenu législatif, elle paraît complètement et nécessairement subordonnée à beaucoup d'autres lois, mais en même temps elle se présente elle-même revêtue de tout le prestige de l'éthique humaniste.

On ne peut s'empêcher de penser que les deux aspects fonctionnent à des niveaux différents : celui de la portée réelle de la loi et celui de ses connotations idéologiques qui lui assurent une véritable aura et qui, en définitive, en masquent le caractère anodin. En même temps qu'elle promulgue certaines contraintes réelles mais secondaires, la Charte des droits de l'Homme reproduit à l'intérieur du discours juridique le langage de l'idéologie dominante. Et c'est peut-être la duplicité même du système juridique dans son entier qui se laisse ici deviner : d'une part le droit se donne, jusque dans l'ambiguïté du mot lui-même, comme indissociablement juridique et éthique, comme institution et comme nature ; mais d'autre part, le discours juridique résiste à toute tentative pour le mettre en relation cohérente et directe avec quelque chose comme la morale.

La duplicité réside peut-être en ceci que cette subordination prétendue du droit à la morale en cache une autre plus réelle et plus déterminante. Les marxistes ici évoqueraient sans doute la non-autonomie du droit par rapport au système économique. Et cette hypothèse, en l'occurrence, paraît fort éclairante : si la place de la Charte des droits de l'Homme dans le système juridique ne se laisse pas clairement circonscrire par une analyse interne du discours juridique, c'est peut-être précisément que ce discours ne trouve pas sa cohérence en lui-même, mais dans son rôle second par rapport au système des relations sociales qu'engendre le processus de production - consommation - échange. S'il en était ainsi en tout cas, le débat entre les «fondamentalistes 》 et leurs adversaires passerait complètement à côté de ce qui est réellement en jeu et se déroulerait tout entier dans un espace idéologique déjà sursaturé.

Université du Québec à Trois-Rivières. 